\title{
An Approach for Intelligent Database Maintenance of HLR and VLR Databases
}

\author{
N. Mallikharjuna Rao, M. M. Naidu, and Seetharam P.
}

\begin{abstract}
Mobile telephony adoption is on the rise, with industry projections suggesting that wireless subscribers will reach 100 billions in India. Recent technological innovations have also dramatically enhanced the capabilities of the wireless telephone; it is one of the most important technologies to track the location of the mobile users. In mobility management basically is performed by two databases, HLR database and VLR database. A mobile user moves anywhere in mobile networks from one LA to another LA in networks, so location registration for call tracking is always needed. An understanding of the issue is some critical to handle with traditional centralized databases. The proposed intelligent database system has the ability to produce good technology in terms of database retrieval and punctual functioning of mobile station systems - technology that is innovative, useful, usable and profitable. Fuzzy Logic plays a major role in extending intelligent database system. It is also proposed to simulate the fuzzy databases.
\end{abstract}

Index Terms-Fuzzy database, location area, HLR, VLR, fuzzy crisp value, fuzzy values.

\section{INTRODUCTION}

In a Personal Communication Services network, the network tracks the locations of the mobile stations (MS) or call transferable so that incoming calls can be delivered to the subscribers. To exercise location tracking, a PCS service area is partitioned into several location areas (LAs). Every LA consists of a group of base stations that communicate with the MSs through radio contact. The major task of mobility management is to update the location of MS when it moves from one LA to another. The location update procedure is referred to as registrations, which is initiated by the MS, the base stations continuously broadcast the corresponding LA addresses to the MSs. When an MS receives a different LA address, it sends a registration message to the network.

The location information is stored in the PCS mobility database is the Home Location Register (HLR) and the Visitor Location Register (VLR). For every LA, there is corresponding VLR. When an MS visits the LA, a temporary record of the MS is created in the VLR to indicate its location (i.e. the LA addresses). For every MS, there is a permanent record stored in the HLR. The record stores the address of the VLR visited by the MS.

The base stations of a LA are connected to a Mobile

Manuscript received June 28, 2012; revised July 25, 2012.

N. Mallikharjuna Rao is with the Annamacharya P.G College of Computer Studies, Rajampet, India (e-mail: drmallik2009@gmail.com).

M. M. Naidu is with the S.V College Engineering, Tirupati, India (e-mail: mmnaidu@yahoo.com).

Seetharam. P is with the Annamacharya Institute of technology and Sciences, Rajampet, India (e-mail: seetharam.p@gmail.com).
Switching Center (MSC), thus, an MSC covers several LAs. One or more MSCs are connected to a VLR, which exchange the location information with VLR through the signaling system Number 7 (SS7) network [1]. Similarly, the VLR communicates with the HLR to exchange the location information using the SS7 messages.

Several approaches have been suggested in the literature for the location management in mobile networks [2]. These are Replication [3], caching, forward pointers; local anchoring and selective paging. Practice has shown that fuzzy databases are more efficient for users with frequently visited locations then all other approaches. Fuzzy database approach is better than all other approaches by its data availability and reliability in the presence of site or communication failures, hence improving the system performance. So we are focusing in this paper with fuzzy databases.

Using a centralized database will not be efficient for handling the high volume of database traffic due to the constant change of user's locations, it will be advantageous to distribute and change user's address at multiple locations. This fuzzy approach makes user profile information more readily available, flexible, easily querying, thus reducing network traffic and database query costs resulting from the registration/de-registration operations that occur when a mobile user moves from one LA to another. This proposed fuzzy database approach has shown its effectiveness for mobile user's with frequently visited locations. Rest of this paper is organized as follows. In section 2 introduce fuzzy logic, section 3 illustrated data structure of HLR and VLR, section 4 covers simulations and concluded in section 5 .

\section{INTRODUCTION TO FUZZY LOGIC}

Fuzzy Logic was introduced [4], [5], [6], [7] as a method for data processing; therein members of a set not only to define certain status of membership or non-membership and also the condition between these two cases. Instead of covering 0 and 1 , fuzzy analyzes from zero to one. In other words, a certain set in Aristotle logic with two members of 0 and 1 is transformed to a set of infinite members with values from 0 to 1 in fuzzy logic and therefore fuzzy logic becomes closer to human's action and thoughts. Fuzzy logic believes that the ambiguity is existed in essence of science and Technology.

Fuzzy logic can solve typical issues and is capable of deploying in both tiny simple microcontroller systems and multi-channel computers, huge network and/or in control systems. Fuzzy logic may be applicable in software, hardware or even combination of both. It is an easy way to 
achieve certain results base on imprecise and ambiguous input data. Fuzzy logic model is based on experience and it relies on user experience till technical understanding of system.

The meaning of fuzzy logic is being paid attention because in the real world many human's reasoning and causes affected by uncertainty and approximation.

Definition of fuzzy set: A fuzzy set upon an original set of ' $x$ ' is a set of pairs

$$
A=\left\{\mu_{A}(x) / x: x \in X, \mu_{A}(x) \in[0,1] \in R\right. \text { in such a }
$$

manner that function of membership degree is called as fuzzy member of A set. Function of membership degree can adopt every real numbers between 0 and 1 .

Characteristics of fuzzy logic are as follows:

1) In fuzzy logic, exact reasoning or normal logic is a special case of approximate reasoning.

2) Every logic system is capable of converting to fuzzy logic

3) In fuzzy logic, knowledge is considered as a set of fuzzy or flexible restrictions on variables.

4) Inference is considered as a process for propagation of these restrictions

5) In fuzzy logic all issues own solutions that show favorableness degree

\section{A. Concepts of Fuzzy Sets}

Introductory citation is essential in order to expand union, intersection, and complement operators in fuzzy set. In choosing union and intersection operators they should be selected in such a manner that for specific cases, exact sets also function correctly, it means that they should own some basic properties like $A \cup U=A, A \cap \phi=\phi, A \cap B=B$ However, for fuzzy sets finding such operators for union and intersection is not possible. Hence, the most common operator for intersection is minimum and for union operator is Maximum. For complement of a fuzzy set the common relation is a minus of membership degree as follows:

$$
\mu_{A}(x)=1-\mu_{A}(x), x \in X
$$

\section{B. Fuzzy Measures}

In this concept regard fuzzy set as possibility distribution that is $\boldsymbol{A}(\boldsymbol{x})$ shows a possibility of specific situation in a set, and with this assumption the first criterion means that there is a possibility that amount $\mathrm{A}$ is equal to amount $\mathrm{B}$, therefore, regarding this interpretation Poss $(A, B)$ is defined as follows:

$$
\left\lfloor\operatorname{Poss}(A, B)=\operatorname{Sup}_{x \varepsilon X}[\min (A(x), B(x))]\right\rfloor
$$

Possibility measure indicates a degree that B is set inside A and is defined as:

$$
\operatorname{Poss}(A, B)=\operatorname{Inf}_{x \varepsilon X}[\max (A(x), 1-B(x))]
$$

\section{Fuzzy Relations}

A classic relation existed on two $X$ and $Y$ sets and a subset of Cartesian product $X^{*} Y$. Therefore, a fuzzy relation of $R$ is a fuzzy set of tuples; each of them has membership degree between 0 and 1 in this relation. If $U$ and $V$ are two continuous sets and $\mathrm{mg}$ : $\mathrm{UxV}$ fuzzy relation of $\mathrm{R}$ is defined as follows:

$$
R=\int_{u x v} \mu R(\mu, v) /(\mu, v)
$$

Hence, one may define operations on fuzzy relations in such an expanded situation like those existed on classic relations.

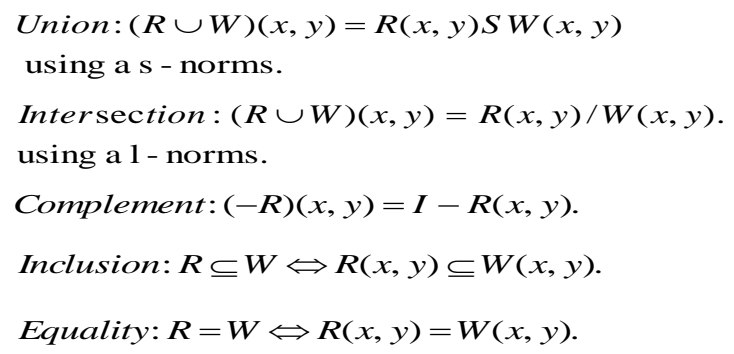

Using the above equations, we can create linguistic and crisp values for any data.

\section{FuZZY DATABASE APPROACHES FOR HLR AND VLR DATABASES}

A database is a computer-based system the purpose of which is to store information regarding a set of entities and to provide users with the capability of organizing, manipulating and retrieving the stored information as requested. Fuzzy database is concerned with the storage and manipulation of knowledge in a manner compatible with human thinking. The database that can accommodate imprecise information can store and manipulate not only precise facts, but also subjective expert opinions, judgment and values that can be specified in linguistic terms.

One of the major concerns in the design of both fuzzy databases is efficiency; this system must be able to perform quickly enough to make interaction with human users feasible, despite large amounts of stored data with their degrees of membership. We describe one of several known approaches to fuzzy databases. Buckles and petry [1982] developed a model for a fuzzy relational database that contains, as a special case, the classical crisp model of a relational database.

The model of a classical relational database consists of a set of multidimensional relations conceptualized as tables. The columns of these tables correspond to fields or attributes and are usually called domains. Each domain is defined on an appropriate domain base (or universe) set.

Let $U$ be a classical set of VLR list, which is having common visited location details called the universe of discourse. An element of $U$ is denoted by $V$. A fuzzy set $\mathrm{F}$ in a universe of discourse $U$ is characterized by a membership function $U_{F}: U \rightarrow[0,1]$ where $\mu_{F}(u)$ for each $u \in U$ denotes the grade of membership of $\mathrm{V}$ in the fuzzy set F. The following the notation used in fuzzy set theory for VLR database is

$$
F=\left\{\mu\left(V_{1}\right) / \mathrm{V}_{1}, \mu\left(V_{2}\right) / \mathrm{V}_{2}, \mu\left(V_{3}\right) / \mathrm{V}_{3}, \ldots . \mu\left(V_{n}\right) / \mathrm{V}_{\mathrm{n}},\right\}
$$




\begin{tabular}{|c|c|c|}
\hline ID & $\begin{array}{c}\text { Common } \\
\text { Visits }\end{array}$ & $\begin{array}{c}\text { Current } \\
\text { visits }\end{array}$ \\
\hline $\mathrm{M}_{1}$ & $3,5,6$ & 4 \\
\hline $\mathrm{M}_{2}$ & $2,3,4$ & 5 \\
\hline $\mathrm{M}_{3}$ & $3,4,5$ & 6 \\
\hline
\end{tabular}

\begin{tabular}{|c|c|c|}
\hline ID & $\begin{array}{c}\text { Common } \\
\text { Visits }\end{array}$ & $\begin{array}{c}\text { Current } \\
\text { visits }\end{array}$ \\
\hline $\mathrm{M}_{1}$ & $3,5,6$ & 4 \\
\hline & & \\
\hline
\end{tabular}

\begin{tabular}{|c|c|c|}
\hline ID & $\begin{array}{c}\text { Common } \\
\text { Visits }\end{array}$ & $\begin{array}{c}\text { Current } \\
\text { visits }\end{array}$ \\
\hline $\mathrm{M}_{1}$ & $3,5,6$ & 4 \\
\hline $\mathrm{M}_{2}$ & $2,3,4$ & 5 \\
\hline & & \\
\hline
\end{tabular}

\begin{tabular}{|c|c|c|}
\hline ID & $\begin{array}{c}\text { Common } \\
\text { Visits }\end{array}$ & $\begin{array}{c}\text { Current } \\
\text { Visits }\end{array}$ \\
\hline $\mathrm{M}_{1}$ & $3,5,6$ & 4 \\
\hline $\mathrm{M}_{2}$ & $2,3,4$ & 5 \\
\hline $\mathrm{M}_{3}$ & $3,4,5$ & 6 \\
\hline
\end{tabular}

VLR database Site 3

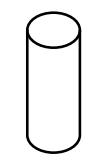

VLR database Site 10

\begin{tabular}{|c|c|c|}
\hline ID & $\begin{array}{c}\text { Common } \\
\text { Visits }\end{array}$ & $\begin{array}{c}\text { Current } \\
\text { Visits }\end{array}$ \\
\hline $\mathrm{M}_{2}$ & $2,3,4$ & 5 \\
\hline $\mathrm{M}_{3}$ & $3,4,5$ & 6 \\
\hline $\mathrm{M}_{4}$ & $4,5,6$ & 3 \\
\hline
\end{tabular}

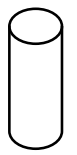

Fig. 1. Frequently visited user's record structures.

Consider that a user $\mathrm{M}_{1}$ has the following set of common visited Location Area's $\{3,5$, and 6$\}$. So we will found a record for user $\mathrm{M}_{1}$ at VLRs of these sites besides the main record at the HLR database. And consider that user $\mathrm{M}_{3}, \mathrm{M}_{4}$, $\mathrm{M}_{5}$ and $\mathrm{M}_{6}$ exits at a site that is not one of its most common sites, so an extra record will be added for these users at the current visited sites.

\section{A. Decision Making of Fuzzy VLR}

As per the fuzzy database concerned, Now we consider during the seven days observation period, the number of roaming users is $m, n, o, p, q, r$, and $s$ on Saturday, Sunday, Monday, Tuesday, Wednesday, Thursday and Friday respectively in MSC service Location area $_{l}$. Then, the observation $S E T$ for each day can be considered as we shown as example follows:

$$
\begin{aligned}
& A=\left\{a_{1}, a_{2}, a_{3}, a_{5}, a_{6}, a_{8}, a_{15} \ldots \ldots \ldots . . a_{m}\right\}, \\
& B=\left\{a_{1}, a_{3}, a_{5} \ldots \ldots \ldots \ldots a_{n}\right\}, \\
& C=\left\{a_{2}, a_{3}, a_{5}, a_{5}, a_{8} \ldots \ldots \ldots \ldots a_{o}\right\}, \\
& D=\left\{a_{1}, a_{3}, a_{5}, a_{6}, a_{7} \ldots \ldots \ldots \ldots a_{p}\right\}, \\
& E=\left\{a_{3}, a_{5}, a_{6}, a_{15}, \ldots \ldots \ldots a_{q}\right\}, \\
& F=\left\{a_{2}, a_{3}, a_{5}, a_{6}, a_{8}, a_{15} \ldots \ldots . a_{r}\right\}, \\
& G=\left\{a_{2}, a_{3}, a_{5}, a_{6}, a_{8}, a_{15} \ldots \ldots a_{s}\right\}:
\end{aligned}
$$

where each set $A, B, C, D, E, F$, and $G$ consists of the visited user's IMSI of Saturday, Sunday, Monday, Tuesday, Wednesday, Thursday, and Friday in the MSC service Location area $_{1}$ respectively.

Thus, the common regularly visited user in $\mathrm{VLR}_{1}$ during the observation period is set $N$ and each of them has been given a FTMSI. And the common set $N$ can be written as follows:

$$
\begin{gathered}
A \cap B \cap C \cap D \cap E \cap F \cap=N \\
N=\left\{a_{3}, a_{5}, a_{6}\right\}
\end{gathered}
$$

Same procedure will continue the $\mathrm{V}_{2}, \mathrm{~V}_{3}$ and $\mathrm{V}_{4}$ as we shown on Fig. 1.

\section{B. Data Structure Representation for HLR and VLR}

A HLR is database of user (subscriber) information, i.e., customer profiles, used in mobile networks. It is a key component of mobile networks such as GSM. A HLR contains user information such as account information, account status, user preferences, features subscribed to by the user, user's current location shown in Fig. 2. The data stored in HLR for the different types of networks is similar but does differ in some details.

A VLR is a database, similar to HLR, which is used by the mobile networks to temporarily hold profiles of roaming users. This VLR data is based on the user information retrieved from the HLR. MSCs use a VLR to handle roaming users.

In HLR database, every record includes two extra fields.

1) ts $=$ the last time of location update

2) $\mathrm{PVLR}=$ the address of VLR where the MS resided at the last check-pointing time

3) $\mathrm{TS}=$ the last check-pointing or back-up time

4) VLR_Counter $=\left\{\left(\mathrm{VLR}_{1}\right.\right.$, Count1 $),\left(\mathrm{VLR}_{2}\right.$, Count 2$) \ldots$ $\left(\mathrm{VLR}_{\mathrm{n}}\right.$, Count $\left.\left._{\mathrm{n}}\right)\right\}$ Where count ' $\mathrm{n}$ ' represents the effective number of MSs entering the $\mathrm{VLR}_{\mathrm{n}}$ during the uncovered period.

5) Note that the VLRs recorded in VLR_Counter are the VLRs in VLR_List*

HLR*
\begin{tabular}{|c|c|}
\hline $\mathrm{MS}$ & $\mathrm{VLR}$ \\
\hline $\mathrm{S}_{1}$ & $\mathrm{~V}_{1}$ \\
\hline $\mathrm{S}_{2}$ & $\mathrm{~V}_{2}$ \\
\hline $\mathrm{S}_{3}$ & $\mathrm{~V}_{3}$ \\
\hline $\mathrm{S}_{4}$ & $\mathrm{~V}_{4}$ \\
\hline
\end{tabular}

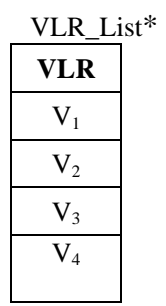

Backup (Non-volatile Storage)
\begin{tabular}{|c|c|c|c|c|c|}
\cline { 5 - 6 } \multicolumn{2}{c}{ HLR } & \multicolumn{2}{c}{ VLR_Counter } \\
\hline $\mathrm{S}_{1}$ & PVLR & ts & VLR & VLR & Count \\
\hline $\mathrm{S}_{2}$ & $\mathrm{~V}_{2}$ & $\mathrm{~T}_{0}$ & $\mathrm{~T}_{2}$ & $\mathrm{~V}_{2}$ \\
\hline $\mathrm{S}_{3}$ & $\mathrm{~V}_{3}$ & $\mathrm{~T}_{3}$ & $\mathrm{~V}_{3}$ & $\mathrm{~V}_{1}$ & 3 \\
\hline $\mathrm{S}_{4}$ & $\mathrm{~V}_{4}$ & $\mathrm{~T}_{4}$ & $\mathrm{~V}_{4}$ & $\mathrm{~V}_{2}$ & 2 \\
\hline $\mathrm{V}_{3}$ & 3 \\
\hline $\mathrm{V}_{4}$ & 4 \\
\hline
\end{tabular}

Fig. 2. Structure of HLR/VLR database.

As per the table I, it will take a wise decision basing which the visiting time will be analyzed as it can also hold the user profiles for infinite days, the word infinite is a mathematical function which as a very good functionality in fuzzy logic databases. Hence a profile is kept for any ' $n$ ' number of days in VLR database and can be brought immediately and increase the VLR_Counter is given in Fig. 2. 
TABLE I: REGISTERED MOBILE RECORDS AT LOCATION AREA

\begin{tabular}{|c|c|c|c|c|c|c|c|c|c|}
\hline $\begin{array}{l}\mathbf{S} \\
\mathbf{N} \\
\mathbf{0}\end{array}$ & $\begin{array}{l}\text { MS } \\
\text { RN }\end{array}$ & $\begin{array}{l}\mathbf{L} \\
\mathbf{A}\end{array}$ & $\begin{array}{l}\text { H } \\
\text { L } \\
\text { R } \\
\text { A } \\
\text { d } \\
\text { d } \\
\text { re } \\
\text { ss }\end{array}$ & $\begin{array}{c}\text { IM } \\
\text { SI } \\
\text { No }\end{array}$ & $\begin{array}{c}\text { Phon } \\
\text { e } \\
\text { No }\end{array}$ & $\begin{array}{c}\text { TMS } \\
\quad \text { I }\end{array}$ & $\begin{array}{c}\text { VL } \\
\text { R } \\
\text { NO }\end{array}$ & $\begin{array}{c}\text { VA } \\
\text { LI } \\
\text { D } \\
\text { TI } \\
\text { E } \\
\text { Fr } \\
\text { om } \\
/ \\
\text { To }\end{array}$ & $\begin{array}{c}\text { TRAN } \\
/ \\
\text { TIME } \\
\text { Start/ } \\
\text { Stop }\end{array}$ \\
\hline 1 & $\begin{array}{c}\text { E16 } \\
4\end{array}$ & $\begin{array}{c}\mathrm{L} \\
1\end{array}$ & $\begin{array}{c}\mathrm{H} \\
1\end{array}$ & $\begin{array}{c}35 \\
93 \\
33 \\
03 \\
49 \\
41 \\
22 \\
1\end{array}$ & $\begin{array}{c}9848 \\
3686 \\
48\end{array}$ & $\begin{array}{c}\text { OAB } \\
\text { CTTT } \\
\text { TT00 } \\
0\end{array}$ & $\mathrm{~V}_{1}$ & $\begin{array}{c}8.0 \\
0 \mathrm{a} \\
\mathrm{m} \\
\text { to } \\
8.0 \\
0 \\
\mathrm{pm}\end{array}$ & $\begin{array}{l}8.00 \mathrm{a} \\
\mathrm{m} \text { to } \\
\infty\end{array}$ \\
\hline 2 & $\begin{array}{c}\text { E16 } \\
4\end{array}$ & $\begin{array}{c}\mathrm{L} \\
2\end{array}$ & $\begin{array}{c}\mathrm{H} \\
1\end{array}$ & $\begin{array}{c}35 \\
48 \\
36 \\
01 \\
95 \\
96 \\
23 \\
7\end{array}$ & $\begin{array}{c}9666 \\
6258 \\
24\end{array}$ & $\begin{array}{c}1 \mathrm{AB} \\
\mathrm{CTTT} \\
\mathrm{T} 000 \\
000\end{array}$ & $\mathrm{~V}_{2}$ & $\begin{array}{c}8.0 \\
0 \mathrm{a} \\
\mathrm{m} \\
\text { to } \\
8.0 \\
0 \\
\mathrm{pm}\end{array}$ & $\begin{array}{c}8.00 \mathrm{a} \\
\mathrm{m} \text { to } \\
\infty\end{array}$ \\
\hline 3 & $\begin{array}{c}\text { E16 } \\
4\end{array}$ & $\begin{array}{c}\mathrm{L} \\
3\end{array}$ & $\begin{array}{c}\mathrm{H} \\
1\end{array}$ & $\begin{array}{c}35 \\
52 \\
31 \\
03 \\
81 \\
99 \\
50 \\
3\end{array}$ & $\begin{array}{c}9966 \\
2583 \\
59\end{array}$ & $\begin{array}{c}\text { Oabct } \\
\mathrm{ttt} 000 \\
0\end{array}$ & $\mathrm{~V}_{3}$ & $\begin{array}{c}8.0 \\
0 \mathrm{a} \\
\mathrm{m} \\
\text { to } \\
8.0 \\
0 \\
\mathrm{pm}\end{array}$ & $\begin{array}{c}8.00 \mathrm{a} \\
\mathrm{m} \text { to } \\
\infty\end{array}$ \\
\hline
\end{tabular}

- $\mathrm{E} 164=+08565(0) 724666$

As per the mobile database concerned, the fuzzy relation solves and handles the frequently visited subscribers at VLR as shown general fuzzy relation is:

$$
A(X)= \begin{cases}l & \text { for } x \in[a, b] \\ l(X) & \text { for } x e(-\infty, a) \\ r(X) & \text { for } x \in(b, \infty)\end{cases}
$$

where $\alpha$ is a function from $(-\infty, a)$ to $[0,1]$ that is monotonic increasing, continuous form the right, and such that $\infty(x)=0$ for $x \in(-\infty, w) ; \alpha$ is a function from $(b, \infty)$ to $[0,1]$ that is monotonic dressing, continues that the lets, and such that $\alpha(x)=0$ for $x \in(w, \infty)$. So, therefore the relation sort the most frequently visited cells and sort the most frequently visited location areas in decreasing order for each user. Decide if this user is a high mobility user $(M=1)$ or low mobility $(M=0)$, so build a user profile as per the information of mobile terminals.

\section{Simulation of FuzZy Database for Mobile TERMINALS}

For representing the mobile database on fuzzy logic is to follow the more meaningful association rule, it is necessary to utilize fuzzy sets over a given database attribute call fuzzy association rule [5], [8]. Given a crisp domain $A$, any arbitrary fuzzy set is defined by a membership function of the form [6] [7], [9].

$$
\mu_{A}: A \rightarrow[0,1]
$$

A fuzzy set may be represented by a meaningful fuzzy label. A reasonable expression of these concepts by trapezoidal membership $A_{1}, A_{2}, A_{3}$ these functions are defined on the interval $[0,80]$. For example, "Low", "Medium", "High" are linguistic variable for fuzzy set frequently visited locations maintained in mobile databases.

$$
\begin{gathered}
\text { Low_Visits }\left(A_{1}\right)=\left\{\begin{array}{l}
1, \quad x \leq 20 \\
(35-x) / 15,20<x<=35 \\
0, x>=15
\end{array}\right. \\
\text { Medium_Visits }\left(A_{2}\right)= \begin{cases}0, & x<20 \text { or } x>=60 \\
(x-20) / 15,20<x<=35 & (60-x) / 15,45 \leq x<60 \\
1, & 35<x \geq 45\end{cases} \\
\text { High_Visits }\left(A_{3}\right)= \begin{cases}0, & x<=45 \\
(18-x) / 155,45<=x<=60 \\
1, & x \geq 60\end{cases}
\end{gathered}
$$

Fig. 3. Triangular membership functions for visits

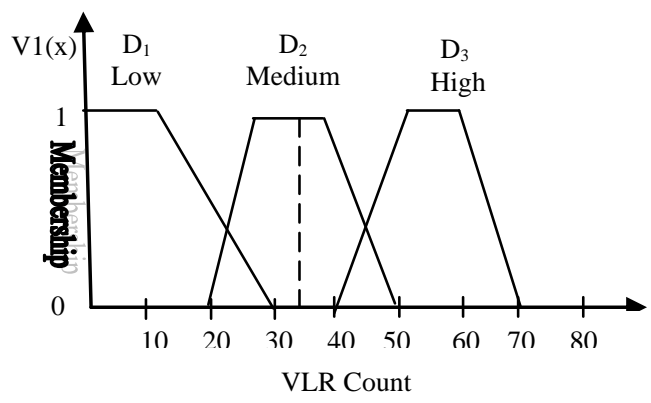

Fig. 4. Crisp values representation for VLR.

A possible discrete approximate, $D_{2}$ of function $\mathrm{A}_{2}$ is show in Fig. 3; its explicit definition in given in Table II. Such approximations are important because they are typical in computer represents of fuzzy sets for frequently visits mobile subscribers. An important concept of fuzzy sets is the concept of and $\alpha$-cut and its variant a strong $\quad \alpha$-cut defined $\alpha^{+} A$.

Fuzzy set $A$ defined on $X$ is $\alpha \in[0,1]$

$$
{ }^{\alpha} A=\{x / A(x)>=\alpha\}, \alpha^{+} A=\{x / A(x)>\alpha\}
$$

The complete characterization of all $\alpha$ - cut and all strong $\alpha$-cuts for the fuzzy sets $A_{1}, A_{2}, A_{3}$,

TABLE II: DiSCRETE APPROXIMATION OF MEMBERSHIP FUNCTION A

\begin{tabular}{|l|c|}
\hline \multicolumn{1}{|c|}{$\mathrm{X}$} & $\mathrm{D}_{2}(\mathrm{x})$ \\
\hline $\mathrm{x} \in(22,24 \ldots 58)$ & 0.0 \\
\hline $\mathrm{x} \in(22,58)$ & 0.13 \\
\hline $\mathrm{x} \in(24,56)$ & 0.21 \\
\hline$\ldots \ldots$ & $\ldots$ \\
\hline $\mathrm{X}(36,38, \ldots 44)$ & 1.00 \\
\hline
\end{tabular}

By function $D_{2}$ of the form: $D_{2}=\{0,2,4 \ldots 80\} \rightarrow[0,1]$

$$
\begin{aligned}
& A_{1}={ }^{0} A_{2}={ }^{0} A_{3}=[0,80]=X \\
& { }^{\alpha} A_{1}=[0,35-15 \alpha],{ }^{\alpha} A_{2}=[15 \alpha+20,60-15 \alpha], \\
& { }^{\alpha} A_{3}=[15 \alpha+45,80] \text { for all } \alpha \varepsilon[0,1], \\
& { }^{\alpha+} A=(0,35-15 \alpha),{ }^{\alpha+} A_{2}=(15 \alpha+20,60-15 \alpha), \\
& { }^{\alpha+} A_{3}=(15 \alpha+45,80) \text { for all } \alpha \varepsilon[0,1] \\
& { }^{1+} A_{1}={ }^{1+} A_{2}={ }^{1+} A_{3}=\phi
\end{aligned}
$$

The set of all levels $\alpha \in[0,1]$ that repress district $\alpha$-cuts of 
given fuzzy set $\mathrm{A}$ is called a level set of A.

Formally

$$
\wedge(A)=\{\alpha / A(x)=\alpha \text { for same } x \in X\}
$$

where $\Lambda$ demotes the level set of fuzzy set $A$ defined on $X$. Example,

$$
\begin{aligned}
& \wedge\left(A_{1}\right)=\wedge\left(A_{2}\right)=\wedge\left(A_{3}\right)=[0.1] \text { and } \\
& \wedge\left(D_{2}\right)=\{0,0.13,0.27,0.4 \ldots .1\} \\
& \Lambda\left(A_{1}\right)=\Lambda\left(A_{2}\right)=\Lambda\left(A_{3}\right)=[0.1] \text { and }
\end{aligned}
$$

In fuzzy set $A$ is the largest membership grade is obtained by any element in the set.

$$
H(A)=\operatorname{Sup}_{X \varepsilon X}(A(x)) .
$$

Therefore, the fuzzy interval "Medium" needs to be explicitly declared outside a relation. i.e. in the programming environment. "Medium" is declared as:

DEFP medium $=(10 / 0.0,20 / 0.13 \ldots . .80 / 1)$ PEND

Where DEFP (define Fuzzy Predicate) is used to define a fuzzy interval and PEND ends the definition. DEFP is part of the data manipulation language in Umano's model. The concept given above might be reducing the tracking time of mobile subscriber, they move from one LA to another. The following results are made using MATLAB software for mobile subscribers.

\section{RESULTS}

The MATLAB is the dominant software package in scientific and mathematical computing and visualization. Originally built to do very fast computations and manipulations of very large arbitrary matrices; now includes things like a scripting language, graphical user interface builder, and extensive mathematical applications. In this paper, we used MATLAB fuzzy Logic toolbox module and implemented the Rule based Fuzzy values as shown in Fig. 5 for $\mathrm{n}$ days and the membership degree values represented as we shown in Fig. 6 and finally presented surface of fuzzy crisp values of mobile subscribers shown in Fig. 7.

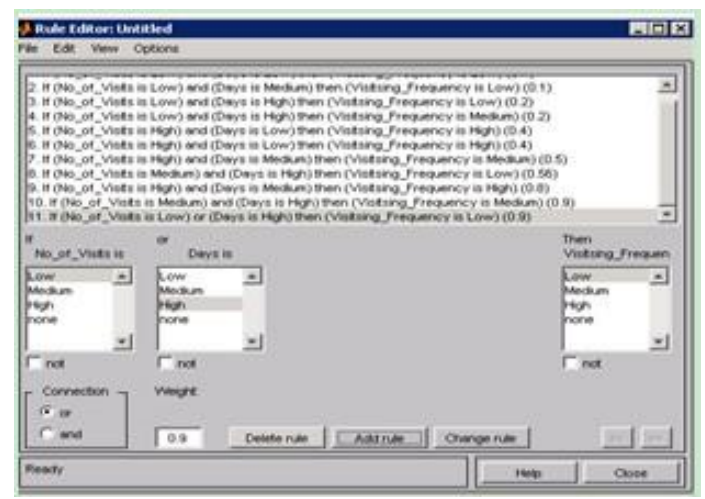

Fig. 5. Rule based fuzzy values for 7 day visits.

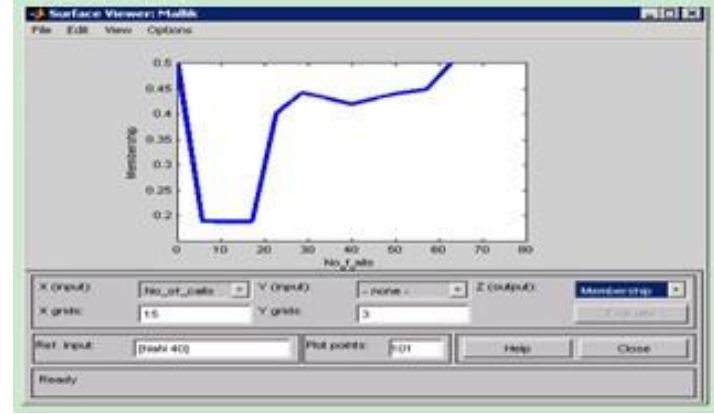

Fig. 6. Report for fuzzy membership values.

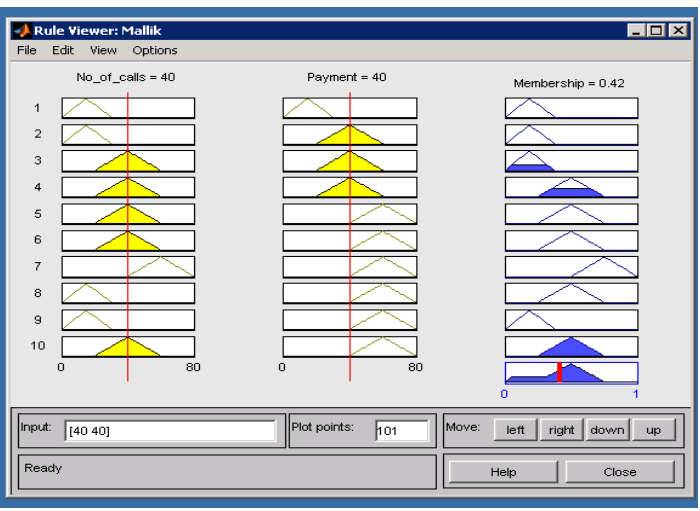

Fig. 7. Fuzzy Surface results for fuzzy crisp

\section{CONCLUSION}

Managing location information of mobile systems is an important issue in mobile computation. The mobile Subscribers are frequently moving between the same set of areas. So the total cost of location management is high. The proposed fuzzy database system is to solve this problem and to reduce the total cost on registration and call delivery algorithms. The proposed fuzzy system work gently and find the habits of the subscriber's profiles.

\section{REFERENCES}

[1] Y. B. Lin and S. K. De Vries, "PCS network signaling using SS7," IEEE Personal Commun. MAg., pp. 44-55, June 1995.

[2] A. Elnahas and N. Adly, "Location management techniques for mobile systems," Information Science. vol.130, pp. 1-22, Elsevier 2000

[3] Y. B Lin, Senior Member, IEEE, "Reduction location update cost in a PCS network," IEEE/ACM Transactions on Networking, vol.5, no.1 February 1997

[4] M. R. Dehghani, M. Abadi, and H. Samavarchi, "The effect of fuzzy logic on database," 2009 World Congress on Computer Science and Information Engineering, IEEE.

[5] R. Intan, "A proposal of fuzzy multidimensional association rules," Journal Informatika vol. 7, no.2 (Terakreditasi SK DIKTI No. 56/DIKTI/Jep/2005). Nov 2006

[6] G. J Klir and B. Yuan, "Fuzzy sets and fuzzy logic: Theory and applications," New Jersey: Prentice Hall, 1995

[7] T. C Ling and K. K Phang, "Fuzzy database framework-relational versus object-oriented model," IEEE, 1997

[8] J. Kacprazyk, "Fuzzy logic in DBMs and querying," Proceedings of the $2^{\text {nd }}$ International conference on Artificial Neural Networks and Expert Systems (ANNES'95), IEEE 1995.

[9] P. Bose and J. kacprzyk, "Fuzziness in database management systems," Springer-Verlag, eds. 1995. 